\title{
Operational Risk Research on Social Pooling Fund Under Diseases Score Settlement System
}

\author{
Zhang Kaijin ${ }^{\text {a, }}$, Wang Min ${ }^{\text {a }}$ \\ ${ }^{a}$ Department of Medical Insurance, School of Public Health, Southeast University, SEU Nanjing, China
}

\begin{abstract}
Objectives: To find out the inner outer risks and its influence on social pooling fund under diseases score settlement (DSS).Methods: To Use step multiple linear regression analysis, the risk factors of the fund have been screened out. The selected risk factors have been taken into BP artificial neural network (BPANN). Results: In 12,724 insured inpatients, chronic diseases accounted for $24.89 \%$. The average medical expense per inpatient was $11,950.88 \mathrm{RMB}$ and per hospitalization expenditure of social pooling fund was 7,665.81RMB. The 10 variables such as age, sex, unit type, hospital level, individual pays, medicine fee, medical fee, operation fee, nurse expense, bed fee and other expense were statistically significant. Conclusion: The growing aging population, changes in disease spectrum, increasing medical costs are all risks of noncontrollable running outside the system. Moral hazard and the defective design of the system belong to the system controllable risks. The results from BPANN were compatible with multiple linear regression analysis.

The payment system plays an important role in health insurance [1]. Good payment can control the hospitalization expenditures in a reasonable scope, while an imperfect one can throw a monkey-wrench into the system. The diseases score settlement (DSS) is payment system of Huai'an in China. This article develops two simple models (step multiple linear regression analysis and back-propagation artificial neural network(BPANN)) to illustrate the risks both inside and outside DSS and explore the risk control function of DSS.

BPANN are the most widely used networks and are considered to be the workhorse of ANNs because of its simplicity and its power to extract useful information from samples [2].Due to its strong learning ability and generalization capability, BP networks have been successfully used in forecasting some financial problems, for example, predicting stock market returns [3], loan risk warning [4] and forecasting bankruptcy firms [5].
\end{abstract}

Index Terms: Diseases score settlement; social pooling fund; multiple linear regression analysis; Backpropagation artificial neural network

(C) 2011 Published by MECS Publisher. Selection and/or peer review under responsibility of the Research Association of Modern Education and Computer Science.

\footnotetext{
* Corresponding author.

E-mail address: njkjzh@163.com
} 


\section{Data and Methods}

\subsection{Data sources and variables}

All the data was obtained from the information system of Huai'an medical insurance settlement center, including information of 12,724 insured inpatients from 2005 to 2008. All research objects were insured inpatient under DSS.

\subsection{Methods}

Multiple linear regression analysis and BPANN were used to analyze the risk factors of social pooling fund.

1) Step multiple linear regression analysis

Hospitalization expenditure of social pooling fund was the dependent variable(Y)and other variables were the metrical determining variables $\left(\mathrm{X}_{i}\right)$, such as age, sex, etc. First, analysis of variance was performed to select independent variables $(p<0.05)$. Second, the variates screened out would be taken into the step multiple linear regression model to fit multiple linear regression (1) and observe the influence of Xi toY using SPSS13.0.

$\mathrm{Y}=\beta_{0}+\beta_{1} X_{1}+\beta_{2} X_{2}+\beta_{3} X_{3}+\cdots$

2) Back-propagation artificial neural network

A neural network is a parallel distributed processing system consisting of an input layer, an output layer, and one or more hidden layers and connected by neurons. Each neuron is a processing element that receives one or more inputs and produces an output signal through a transfer function (activation function)[6]. We set up a standard three-layer BPANN model to predict the hospitalization expenditure of social pooling fund by the use of Matlab 7.0.The model parameters (connection weights and node biases) are adjusted iteratively by a process of minimizing the estimation error function. Basically, the final output of the BPANN-based forecasting model can be represented as

$$
y=f(x)=\alpha_{0}+\sum_{j=1}^{q} w_{j} \varphi\left(\alpha_{j}+\sum w_{i j} x_{i}\right)
$$

Where $\mathrm{Xi}=(\mathrm{i}=1,2, \cdots, \mathrm{p})$ represents the input patterns or independent variables, $\mathrm{f}(\mathrm{x})$ or $\mathrm{y}$ is the output or dependent variable, $\alpha \mathrm{j}(\mathrm{j}=0,1,2, \cdots, \mathrm{q})$ is a bias on the $\mathrm{jth}$ unit, $w_{i j}(i=1,2, \cdots, p ; j=1,2, \cdots, q)$ is the connection weight between layers of the model, $\varphi(\bullet)$ is the transfer function of the hidden layer, $p$ is the number of input nodes,$q$ is the number of hidden nodes and $\mathrm{n}$ is the number of output nodes.Fig 1 shows the neural network structure .First, 1000 samples were randomly sampled from 12,724 inpatients and then, randomly be divided into two parts, $70 \%$ of the samples as neural network training samples, and $30 \%$ reserved for testing samples. Second, Significant factors selected by Multiple linear regression analysis would be put into the model as input neuron $\mathrm{X}_{i}$, and the hospitalization expenditure of the social pooling fund $Y$ as output neuron to simulate the training sample trend. Through the simulation of neural network model, the trend of testing sample had been predicted. In addition, before the start of the model training, samples should firstly been normalized using $\operatorname{premnmx}(P, T)$ function to eliminate influence of dimension.

\section{The operation of hospitalization social pooling fund and risk factor screening}

\subsection{Status of the insured inpatients who use the fund}

There were 12,724 insured inpatient from 2005 to $2008.41 .35 \%$ were over the age of 60 . Among them, chronic diseases, such as hypertension, diabetes, coronary disease, cerebral infarction, etc. accounted for 24.89\%; The average medical expense per inpatient was 11,950.88RMB and per hospitalization expenditure of 
social pooling fund was $7,665.81 \mathrm{RMB}$. The proportion of the social pooling fund expenditure occupied $63.41 \%$ of the total medical expenditure.

One-way analysis of variance

Taking the hospitalization expenditure of the social pooling fund $(\mathrm{Y})$ as response variable ,age ,sex, hospital level and other study variables were taken into One-way analysis. The variables such as age,sex, unit type,hospital level, individual pays , medicine fee, medical fee, operation fee, nurse expense, bed fee and other expense were statistically significant.

\section{Step multiple linear regression analysis}

We took 10 factors $(\mathrm{p} \leqslant 0.05)$ selected by one-way analysis into the model as independent variables and the hospitalization expenditure of social pooling fund $(\mathrm{Y})$ as response variable. Standardized regression coefficients, significance level and coefficient of determination( $R$ square) had been used to evaluate the influence degrees of the main factors on Y. Inclusion criteria of the step regression model was 0.05 and exclusion criteria was 0.10 .

The fitness of the model was accepted, with the exception of unit types, all others were significantly associated with the hospitalization expenditure of social pooling fund. The variables included in the model can explain $83.2 \%$ changes of $\mathrm{Y}\left(\mathrm{R}^{2}=0.832\right)$. Variable medicine fee has the biggest influence on $\mathrm{Y}$, whose Standardized Coefficients is 0.64.Individual pays has a negative influence on $Y$. The more individual pays, the less hospitalization expenditure of the social pooling fund $(\mathrm{Y})$ spends. The fund paid more for male than for female. The older patients, the higher fund expenses. Second-tier hospitals had been paid more than tertiary hospitals. The average of social pooling fund in second-tier hospitals was 9,560.87 Yuan RMB, which was higher than tertiary hospitals'.

Multivariate regression equation is as follows.

$Y=783.47+0.59 X_{1}+0.51 X_{2}+0.56 X_{3}+1.43 X_{4}-0.54 X_{5}+0.83 X_{6}$ The order of factors influence on

$+295.06 X_{7}+2.95 X_{8}-117.01 X_{9}-126.01 X_{10}+741.85 X_{11}$

hospitalization expenditure of the social pooling fund $\mathrm{Y}$ is as follows.

Medicine fee $>$ medical fee $>$ other expense $>$ individual pays $>$ bed fee $>$ operation fee $>$ age group $>$ hospital level $>$ nurse expense $>$ sex (Table1).

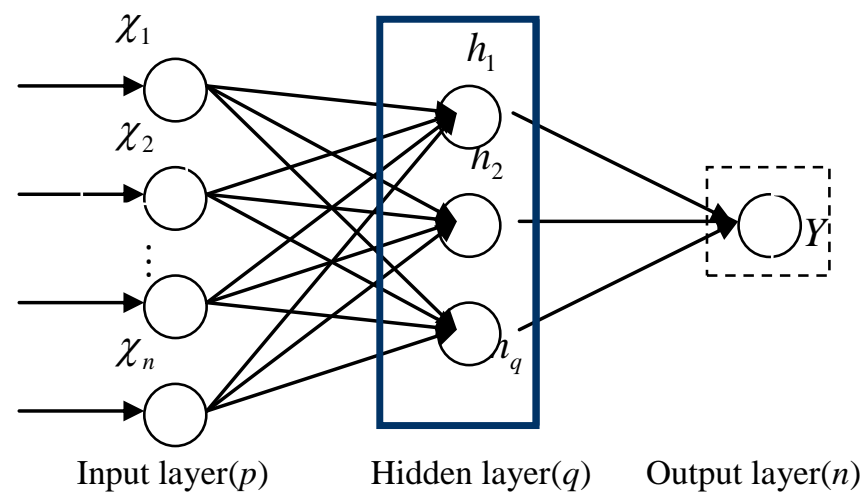

Fig. 1. BPANN Forecasting Model of the hospitalization expenditure of the risk-pooling fund 
Table 1 Step multiple linear regression model fitness

\begin{tabular}{ccccccc}
\hline \multicolumn{7}{c}{ Unstandardized } \\
variables \& their code & \multicolumn{2}{c}{ Coefficients } & $\mathrm{SC}^{\mathrm{b}}$ & $t$ & $p$ \\
\hline constant & $\beta_{0}$ & 783.47 & 99.84 & & 7.85 & $<0.05$ \\
medicine fee & $X_{1}$ & 0.59 & 0.01 & 0.64 & 75.72 & $<0.05$ \\
medical fee & $X_{2}$ & 0.51 & 0.01 & 0.32 & 55.16 & $<0.05$ \\
other expense & $X_{3}$ & 0.56 & 0.01 & 0.28 & 54.63 & $<0.05$ \\
bed fee & $X_{4}$ & 1.43 & 0.05 & 0.16 & 27.14 & $<0.05$ \\
Individual pay & $X_{5}$ & -0.54 & 0.02 & -0.26 & -24.44 & $<0.05$ \\
operation fee & $X_{6}$ & 0.83 & 0.03 & 0.11 & 26.51 & $<0.05$ \\
age group & $X_{7}$ & 295.06 & 19.88 & 0.06 & 14.84 & $<0.05$ \\
nurse expense & $X_{8}$ & 2.95 & 0.57 & 0.02 & 5.17 & $<0.05$ \\
sex & $X_{9}$ & -117.01 & 59.80 & -0.01 & -1.96 & 0.05 \\
hospital level & $X_{10}$ & -126.01 & 163.63 & 0.00 & -0.77 & 0.44 \\
hospital level & $X_{11}$ & 741.85 & 77.44 & 0.04 & 9.58 & $<0.05$ \\
\hline
\end{tabular}

\subsection{The application of BPANN on trend prediction of hospitalization expenditure of the social pooling fund}

Introducing BPANN into trend prediction of hospitalization expenditure of the social pooling fund can verify the effectiveness of factors selected by multiple linear regression model and play an important role in

evaluating and testing the predictions of previous research.10 factors selected by multiple linear regression had been taken into BPANN as input variables and hospitalization expenditure of the social pooling fund $Y$ as output variable. to establish the network(Model parameters: show $=50$; epochs=1000; goal=1e$3 ; \mathrm{l}=0.1) . \mathrm{X}_{\mathrm{i}}=(\mathrm{i}=1,2, \cdots \mathrm{n})$ in testing samples had been taken into the trained neural network to predict the hospitalization expenditure of the social pooling fund(Y).

Figure 2 shows the forecasting effects of testing samples. In Figure2,a cross is the symbol for the actual hospitalization expenditure of the social pooling fund of testing samples, and every small circles represent predicted value. Thus it can be seen from the two figures below that the forecasted value was nearly consistent with the real value, and relative error had been controlled between 0 Yuan to 1.5 Yuan after anti-normalization (Figure3).

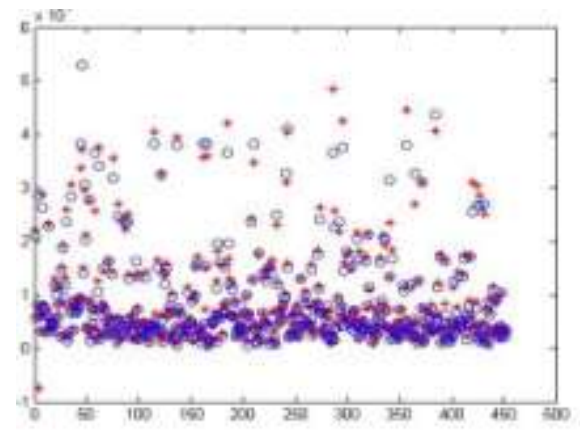

Fig.2 The prediction results of testing samples 


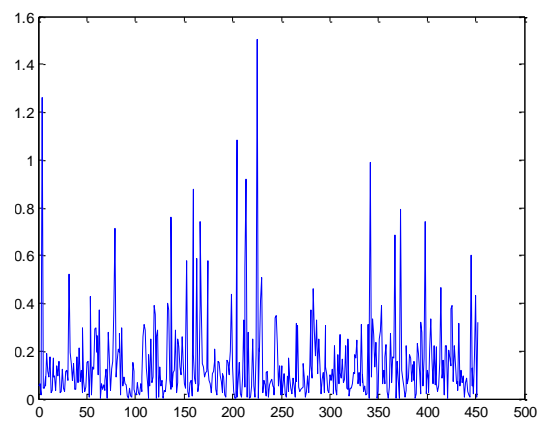

Fig.3 The relative error of prediction of testing samples

\section{Discussion}

Health insurance faces risks both outside and inside the system. The former is caused by social environment of health insurance such as politics, economy and culture etc. It is non-controllable and generally hard to avoid. The growing aging population, changes in disease spectrum, increasing medical costs resulted by "High, new, sharp" medical equipment, technology and medicine are all risks of non-controllable running outside the system. The latter generates in the process of operation system and has roots in the absence of supervision mechanism or the defects and man-made risks in system design and practical operation[7].Both the risks outside the system and inside are all difficult to completely eliminate ,but they can be reduced through system improvement, and this process is called "risk institutionalization"[8].Establishing health insurance system to reduce the loss caused by illness risks or carrying out DSS to prevent healthcare providers from refusing patients and decomposing hospital treatment are all channels of "risk institutionalization".

A multiple linear regression equation which assumes the expenditure of the social pooling fund as the dependent variable $(Y)$, other factors as variables $(X)$ have been get up. The results from BPANN were compatible with multiple linear regression analysis.The 10 variables such as age, sex, unit type, hospital level, individual pays, medicine fee, medical fee, operation fee, nurse expense, bed fee and other expense were the risk factors of hospitalization expenditures of social pooling fund.

In summary, the growing aging population, changes in disease spectrum, increasing medical costs resulted by "High, new, sharp" medical equipment[9]-[10], technology and medicine are all risks of non-controllable running outside the system[11]. Moral hazard[12], the defective design of the system and the problem of diagnosis escalation belong to the system controllable risks. Some measures can be taken to reduce the risk influence on social pooling fund, such as establishing and perfecting clinical pathway to standardize the medical service, setting up the index system of medical insurance and improving the adjustment mechanism of diseases scores, etc.

\section{References}

[1]Chen Yanlin, Liu Hongmin \& Zhang Chen. A comparative study on payment for medical expenses at home and abroad. Chinese Chongqing Medical Journal, Vol.37, No.9 (Mar., 2008), pp. 993-995(in Chinese) [2]Li, Qiang, Yu, Jing-Yuan, Mu, Bai-Chun, \& Sun, Xu-Dong (2006). BP neural network prediction of the mechanical properties of porous NiTi shape memory alloy prepared by thermal explosion reaction. Materials Science and Engineering: A, 419(1-2):214-217.

[3]Enke, David, \& Thawornwong, Suraphan.The use of data mining and neural networks for forecasting stock market returns. Expert Systems with Applications, 29(4),2005,pp.927-940. 
[4]Yang, Baoan, Li, Ling X., Ji, Hai, \& Xu, Jing. An early warning system for loan risk assessment using artificial neural networks. Knowledge-Based Systems, 14(5-6),2001,pp.303-306.

[5]Lee, Kidong, Booth, David, \& Alam, Pervaiz.A comparison of supervised and unsupervised neural networks in predicting bankruptcy of Korean firms. Expert Systems with Applications, 29(1),2005,pp.1-16.

[6]Lee, Kidong, Booth, David, \& Alam, Pervaiz (2005). A comparison of supervised and unsupervised neural networks in predicting bankruptcy of Korean firms. Expert Systems with Applications, 29(1):1-16

[7]Project Opening Report of Research on Medical Insurance Payment Management and Risk Prevention ,2005, http://www.mib.com.cn

[8]Lean Yu,Shouyang Wang \& Kin Keung Lai.A multiscale neural network learning paradigm for financial crisis forecasting, Neurocomputing,

www.elsevier.com/locate/neucom

[9]WHO. Updated projections of global mortality and burden of disease, 2002-2030: data sources, methods and result s.Colin D.Math2ers, Dejan loncar evidence and information for policy working paper evidence and information for policy World Health Organization.Geneva: WHO, 2005: 10

[10]Ming-Jye Wang, Shu-Ping Lin.Study on doctor shopping behavior:Insight from patients with upper respiratory tract infection in Taiwan. Health Policy.Vol.94, Issue 1(2010):61-67

[11]Yang Xuedong. Risk Society and Order Restoration, Chinese Society Science Document Publishing House, pp.23-pp.133

[12]Wu Chuanjian.Analysis on Moral Hazard and fund safety of medical insurance in China.Chinese Health Economics, Vol.24, and No.11 (Nov., 2005):47-48(in Chinese) 